\title{
Vibration Control on Multilayer Cable Moving through the Crossover Zones on Mine Hoist
}

\author{
Xia Peng, ${ }^{1,2}$ Xian-sheng Gong, ${ }^{1}$ and Jin-jun Liu ${ }^{3}$ \\ ${ }^{1}$ College of Mechanical Engineering, Chongqing University, Chongqing 400044, China \\ ${ }^{2}$ Mechanical and Electrical Engineering College, Shihezi University, Shihezi, Xinjiang 832003, China \\ ${ }^{3}$ CITIC Heavy Industries Co., Luoyang 471039, China \\ Correspondence should be addressed to Xian-sheng Gong; cqxsgong@cqu.edu.cn
}

Received 12 May 2015; Revised 10 November 2015; Accepted 11 November 2015

Academic Editor: Toshiaki Natsuki

Copyright (C) 2016 Xia Peng et al. This is an open access article distributed under the Creative Commons Attribution License, which permits unrestricted use, distribution, and reproduction in any medium, provided the original work is properly cited.

\begin{abstract}
Mine hoist is an important piece of equipment in mine hoist systems, and we achieve deep mine hoist through the multilayer winding, but the cable always undergoes severe shock and vibration during the winding process, and the dynamic load and wear would greatly reduce the lifetime of the cable and cause potential safety hazard. In this paper, we start from the course of crossing over of winding cable, use the methods of differential geometry, mechanics, and mathematical analysis, study the movements of the crossover, and derive the important formula that can reduce the vibration of cable during the course of crossover: the formula about central angle of the crossover arc. The results display that four factors contribute to central angle of the crossover arc, that is, the gap of the rope grooves, friction coefficient of the cable, and diameter of the drum and the cable. The result can provide valuable information for designing multilayer winding mine hoist.
\end{abstract}

\section{Introduction}

Along with the exploitation of the deep earth resources, the deep mine hoisting is more and more important. Thus, the most common winding system is the multilayer winding drum installations in mine hoisting applications. Then the external wear and plastic deformation are serious at the flange of the drum and the crossover zone [1]. After a long period of research, the major factors that affect multilayer winding are these: the type and arrangement form of the groove, the way and the parameters that the riser and filler rings afford, the way the cable moves through the crossover zone, central angle of the crossover arc, the velocity and the accelerated velocity of the hoisting, the numerical value of the cable's fleet angle, the structure of the cable, operation and maintenance of the cable, and so forth. These factors play an important role in lifetime, vibration, and arrangement of the cable [2].

The winder drum surface is covered by parallel circular grooves with two diametrically opposed crossover zones per drum circumference, and the mechanism applied on the winder drum surface in order to achieve a uniform coiling pattern, as shown in Figure 1. Each zone offsets the grooves by half a cable diameter until the cable reaches the drum flange. Then riser and filler rings force a layer change, and the spooling continues in the opposite direction. But it is difficult to install a certain device to reduce the cable's vibration, extrusion, and wear when moving through crossover zones [3]. The structure of the riser and filler rings is connected with central angle of the crossover arc as shown in Figure 1, and the stability of the cable is of great importance when it is moving through the crossover zone; the stability can affect the lifetime, vibration, and orderly arrangement of the rope. So it is necessary to study the rational parameter of the crossover zone.

At present, the problem of the crossover transition in multilayer winding is mainly on the way of differential geometry and mathematical analysis. Then the study is mainly about the fiber coiling onto the solid of revolution. In the technology of fiber's coiling, the geodesic is adopted in coiling in the early stage because of steady and simple calculating. He [4] gave the basic equation of the fiber coiling onto the solid of revolution along geodesic, and the equation must meet the conditions of not bumping and complete winding. Known from the differential geometry, the geodesic is the most stable 


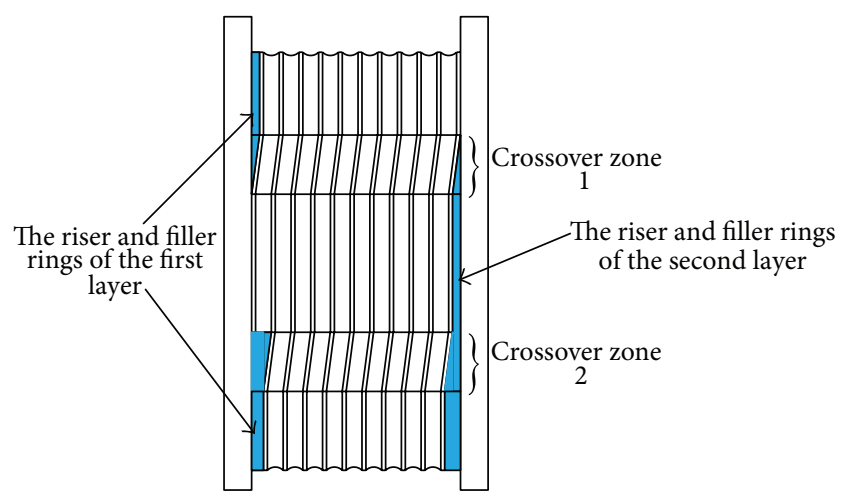

FIgURE 1: Expanded view of the drum with parallel groove.

position on surface. So the position of geodesic is the most stable one and does not slip. If the fiber coils diverge from the geodesic, it will slide to the geodesic. However, the precondition that the theory of the stable winding along the geodesic is correct is discarding the friction. Leng [5] gave several stability equations of the fiber coiling onto several solids of revolution along nongeodesic, but he did not give the equation of coiling onto the torus. Menges et al. [6] (see also De Carvalho et al. [7] and $\mathrm{Zu}$ et al. [8]) published their new idea in the 33rd Technology Anniversary of SPI; the new idea claims that the fiber could coil along the nongeodesic without slide under the condition of friction. The condition of stable coiling along the nongeodesic is that

$$
\frac{K_{g}}{K_{n}} \leq \mu,
$$

where $K_{g}$ denote geodesic curvature, $K_{n}$ denote normal curvature, and $\mu$ denote friction coefficient. But the original text did not give the course of derivation; they only showed the photos of the successful experiment. Wells and McAnulty [9] and Scholliers and Van Brussel [10] mentioned that they achieve the steady coiling along the nongeodesic. Li and Lin [11] supposed (1) is correct; then they derived the differential equation of the fiber coiling onto the solid of revolution along the nongeodesic. Fu et al. [12] derived the equation of the fiber coiling along the nongeodesic and gave the boundary condition.

The friction exists between the cable and the drum or among the cables of different layers, and this kind of friction could resist a certain degree of slip. So the cable could remain stable even though it diverges from the geodesic within limits, and we can derive the equation of the crossover curve with the theory of stable coiling along the nongeodesic. Gong et al. [13] analyzed the course of the cable winding on the drum which installed a single crossover groove; differential geometry relation of the crossover zones was investigated. Reasonable length of crossover zone is derived so as to lead the cable move steadily from one groove to another, and then the vibration, extrusion, and wear were reduced. But in order to calculate expediently, the helical angle of the firstlayer rope in crossover zone was supposed to be zero, and the mathematics model of the first layer was too complex, and it was disadvantageous to calculate.

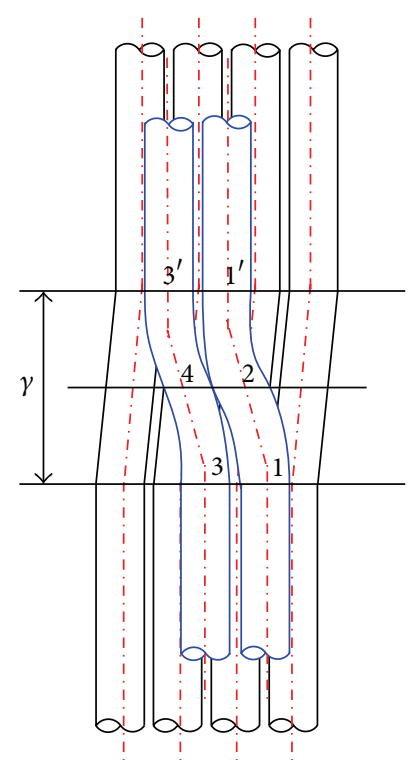

Figure 2: Plan figure of the crossover.

In this paper, we will utilize the theory of the fiber stable coiling onto the solid of revolution along the nongeodesic and use the thinking and methods of Gong et al. [13] and then improve their mathematic model and computing method and derive the more reasonable computational equation of the length of crossover.

\section{The Derivation of Theoretical Equation}

When one selects the diameter of the drum, the bending stress of the cable should not be large, and then the cable could keep a certain carrying capacity and lifetime. When the ratio of $D / d$ is greater than 80 and the numerical value of $D$ is larger than $1200 \delta$ ( $\delta$ denote the diameter of one steel wire of the cable, $d$ denote the diameter of the cable, and $D$ denote the diameter of the drum), the bending stress that the cable bears is not enough to affect the lifetime of the cable, so the rigidity of the cable in the bending position can be ignored. In order to make the study have universal significance, the cable winding onto the drum with parallel circular grooves is supposed, as shown in Figure 1.

\subsection{Differential Geometry Relation of the Cable of Crossover} Zones. In order to derive the reasonable length of crossover zone, the differential geometry relationship between the upper layer and the under layer in the crossover zone must be clear. The following fundamental assumptions are made: (1) the cross section of the cable is circle; (2) the cable's material is uniform; (3) there is no compressional deformation between the upper layer and the under layer. The plan figure and sectional view of the crossover are showed as Figures 2 and 3. In order to research conveniently, a certain cable of crossover zone is selected for study and the graphic model of the cable is drawn as Figure 4 . When the cable moves from one groove to another within the crossover zone, a contact curve is formed 


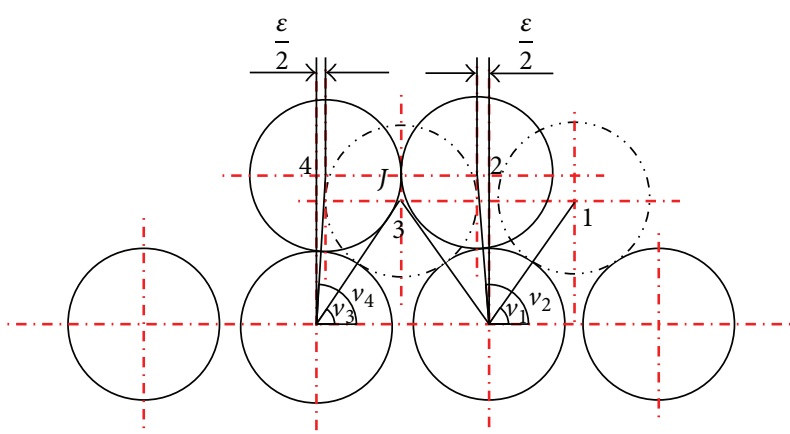

FIGURE 3: Sectional view of the crossover.

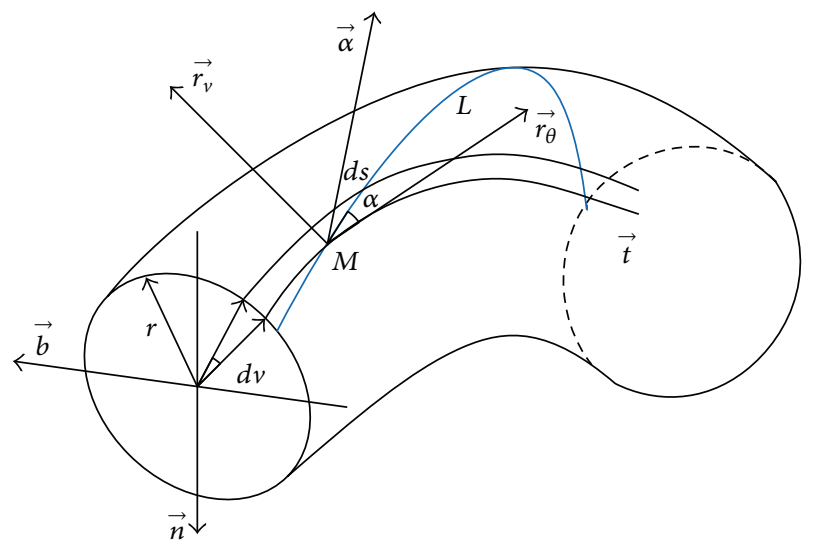

FIGURE 4: The graphic model of the cable of crossover.

between the cable of the first layer and the second layer. The shape of the contact curve is the same as the generatrix and axis of the cable. The curve $L$ is supposed as the axis of the cable of the second layer, and the angle that the first layer makes with the second layer is named as $\alpha$; the included angle between the horizontal axis of the second layer and the first layer is named as polar angle $v$. When the drum of the hoist rotates an angle element $d \theta$, the arbitrary point $M$ in the curve $L$ will move forward arc element $d s$, and then the arbitrary point $M$ rotates an angle element $d v$ from the polar angle $v$. The arc element $d s$ is projected in tangential direction and vertical direction of the generatrix of the first-layer rope; then two relations can be derived as follows:

$$
\begin{aligned}
& d s \cos \alpha=(R+r+r \sin v) d \theta, \\
& d s \sin \alpha=r d v .
\end{aligned}
$$

The two formulas can be linearized (ignore the infinitesimal higher than the second order) and get the differential geometry relation of crossover as shown in

$$
d \theta=\frac{r}{R+r} \frac{1}{\tan \alpha} d v
$$

2.2. Geometrical Parameter of Crossover Zone. As shown in Figures 2, 3, and 4, the cable of the first layer moves from cross section 1 and achieves position 2 at the top of the first layer and then moves down and achieves position $1^{\prime}$ and completes once crossover transition. The cable keeps on coiling along the groove formed from the first layer and achieves position 3 and then begins another crossover transition. The two cables contact at the position $J$ as shown in Figure 2. The cable will produce an enormous extrusion force in the contact point if the geometric parameter of crossover is unreasonable and also produce slippage and shock in declining stage during position $2-1^{\prime}$ and position $4-3^{\prime}$ and then produce vibration and excessive wear. In order to eliminate or alleviate the above-mentioned bad effects, reasonable crossover length should be derived to ensure that the two cables deviate the same distance on the top of the first layer at the contact point $J$, and the distance should be $\varepsilon / 2$, where $\varepsilon$ denote the gap of the groove and $\gamma$ is the angle defining the central angle of the crossover arc. Thus the two cables could keep contact and make the extrusion force minimum. Equations (4)-(6) can be derived from geometrical relationship in Figures 2 and 3:

$$
\begin{aligned}
& v_{1}=v_{3}=\frac{\pi}{2}-\arcsin \frac{(d+\varepsilon) / 2}{d}=\arccos \frac{d+\varepsilon}{2 d} \\
& v_{2}=\frac{\pi}{2}+\arcsin \frac{\varepsilon}{2 d} \\
& v_{4}=\frac{\pi}{2}-\arcsin \frac{\varepsilon}{2 d} .
\end{aligned}
$$

Equation (7) can be derived through simultaneous equations (3)-(6):

$$
\gamma=2 \int_{\arccos ((d+\varepsilon) / 2 d)}^{\pi / 2} \frac{r}{R+r} \frac{1}{\tan \alpha} d v .
$$

Equation (7) is the differential geometry relationship of crossover and also is the mathematical expression of the central angle of the crossover arc. The function relationship between parameter $\alpha$ and parameter $v$ should be made sure of before solving the integral.

2.3. Reasonable Condition of the Crossover Parameter. When the cable of the second layer moves from one groove to another through the top of the first-layer rope, the cable of the second layer is in balance under the action of tension, friction, and extruding force, as shown in Figure 1. In order to study expediently, Figure 4 is flipped and drawn as Figure 5. The hook face is named as $\Sigma_{1}$. In order to find out the function relationship between parameter $\alpha$ and parameter $v$, the differential geometry relationship of the curve $L$ at the point $M$ is acquired based on the theory of differential geometry and mechanics, that is, the relationship between geodesic curvature $K_{g}$ and normal curvature $K_{n}$ of the curve $L$ at the point $M$ :

$$
K_{g}=-K_{n} \mu
$$

In the formula $\mu=\tan \varphi$, the parameter $\mu$ is the coefficient of sliding friction. The equation is consistent with (1).

The curved surface equation determines the geodesic curvature $K_{g}$ and normal curvature $K_{n}$ of the curve $L$ in the hook face. In order to find out the function relationship between parameter $\alpha$ and parameter $v,(8)$ must be solved. 


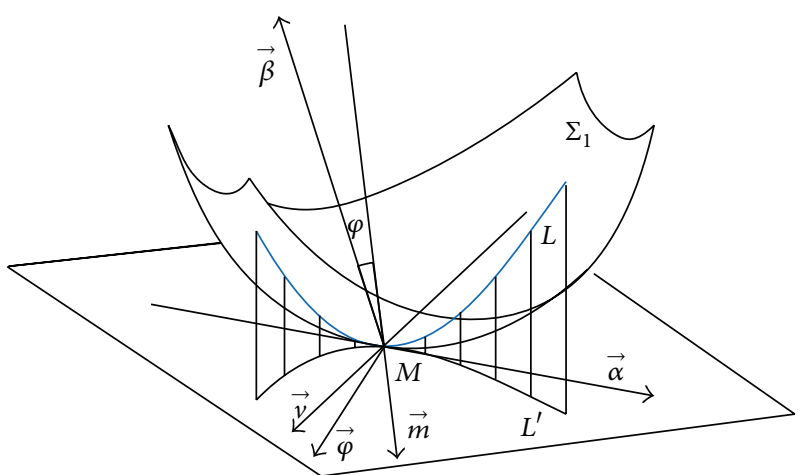

FIGURE 5: The relational graph on the geodesic curvature $K_{g}$ and normal curvature $K_{n}$ of the contact curve at the point $M$.

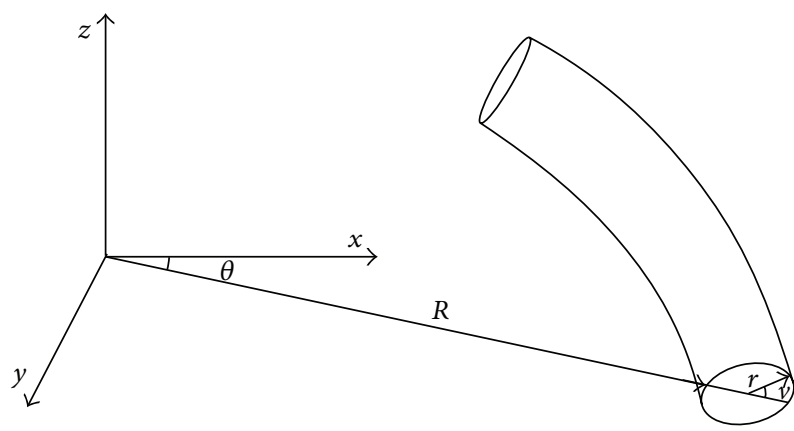

FIGURE 6: The sketch map of each parameter of the torus.

2.4. The Equation of the Hook Face $\Sigma_{1}$. The cable of the first layer winds on the drum which installed parallel groove with two crossover zones, and the cable only shifts a half diameter of one cable along axis, and the helical angle is very small and can be looked at as zero, so the cable of the first layer can be looked at as torus approximate. The parameter equation of the torus can be derived through Figure 6:

$$
\begin{aligned}
& x=(R+r+r \cos v) \cos \theta, \\
& y=(R+r+r \cos v) \sin \theta, \\
& z=r \sin v
\end{aligned}
$$

where $\theta$ denote the central angle that corresponds to the drum at optional position in the torus and $v$ denote the polar angle of two-layer cable.

The hook face $\Sigma_{1}$ can also be denoted as $r=r(\theta, v)$; the Liouville equation is given by Gray [14]:

$$
K_{g}=\frac{d \alpha}{d s}-\frac{1}{2 \sqrt{G}} \frac{\partial \ln E}{\partial v} \cos \alpha+\frac{1}{2 \sqrt{E}} \frac{\partial \ln G}{\partial \theta} \sin \alpha
$$

The Euler equation is given as follows:

$$
K_{n}=\frac{I I}{I}=\frac{L}{E} \cos ^{2} \alpha+\frac{2 M}{\sqrt{E G}} \cos \alpha \sin \alpha+\frac{N}{G} \sin ^{2} \alpha,
$$

where

$$
\begin{aligned}
& E=\vec{r}_{\theta} \cdot \vec{r}_{\theta}=\left|\frac{\partial x}{\partial \theta}\right|^{2}+\left|\frac{\partial y}{\partial \theta}\right|^{2}+\left|\frac{\partial z}{\partial \theta}\right|^{2}, \\
& F=\vec{r}_{\theta} \cdot \vec{r}_{v}=\left|\frac{\partial x}{\partial \theta}\right|\left|\frac{\partial x}{\partial v}\right|+\left|\frac{\partial y}{\partial \theta}\right|\left|\frac{\partial y}{\partial v}\right|+\left|\frac{\partial z}{\partial \theta}\right|\left|\frac{\partial z}{\partial v}\right|, \\
& G=\vec{r}_{v} \cdot \vec{r}_{v}=\left|\frac{\partial x}{\partial v}\right|^{2}+\left|\frac{\partial y}{\partial v}\right|^{2}+\left|\frac{\partial z}{\partial v}\right|^{2} \\
& D=\sqrt{E G-F^{2}} \text {, } \\
& L=\frac{\left(\vec{r}_{\theta \theta}, \vec{r}_{\theta}, \vec{r}_{v}\right)}{D}=\frac{1}{D}\left|\begin{array}{lll}
\frac{\partial^{2} x}{\partial \theta^{2}} & \frac{\partial^{2} y}{\partial \theta^{2}} & \frac{\partial^{2} z}{\partial \theta^{2}} \\
\frac{\partial x}{\partial \theta} & \frac{\partial y}{\partial \theta} & \frac{\partial z}{\partial \theta} \\
\frac{\partial x}{\partial v} & \frac{\partial y}{\partial v} & \frac{\partial z}{\partial v}
\end{array}\right| \\
& M=\frac{\left(\vec{r}_{\theta v}, \vec{r}_{\theta}, \vec{r}_{v}\right)}{D}=\frac{1}{D}\left|\begin{array}{ccc}
\frac{\partial^{2} x}{\partial \theta \partial v} & \frac{\partial^{2} y}{\partial \theta \partial v} & \frac{\partial^{2} z}{\partial \theta \partial v} \\
\frac{\partial x}{\partial \theta} & \frac{\partial y}{\partial \theta} & \frac{\partial z}{\partial \theta} \\
\frac{\partial x}{\partial v} & \frac{\partial y}{\partial v} & \frac{\partial z}{\partial v}
\end{array}\right|, \\
& N=\frac{\left(\vec{r}_{v v}, \vec{r}_{\theta}, \vec{r}_{v}\right)}{D}=\frac{1}{D}\left|\begin{array}{lll}
\frac{\partial^{2} x}{\partial v^{2}} & \frac{\partial^{2} y}{\partial v^{2}} & \frac{\partial^{2} z}{\partial v^{2}} \\
\frac{\partial x}{\partial \theta} & \frac{\partial y}{\partial \theta} & \frac{\partial z}{\partial \theta} \\
\frac{\partial x}{\partial v} & \frac{\partial y}{\partial v} & \frac{\partial z}{\partial v}
\end{array}\right|
\end{aligned}
$$

$K_{g}$ and $K_{n}$ are solved according to (10)-(12), and the results can be simplified as follows:

$$
\begin{aligned}
& K_{n}=-\frac{(R+r) \sin ^{2} \alpha+r \cos v}{r(R+r+r \cos v)}, \\
& K_{g}=\frac{d \alpha}{d v} \frac{\sin \alpha}{2 r}+\frac{\cos \alpha \sin v}{R+r+r \cos v} .
\end{aligned}
$$

The results which had been simplified are substituted into differential equation (7), and then differential equation (14) can be gotten:

$$
\begin{aligned}
& \frac{d \alpha}{d v} \frac{\sin \alpha}{2 r}+\frac{\cos \alpha \sin v}{R+r+r \cos v} \\
& \quad=\frac{\mu(R+r) \sin ^{2} \alpha}{r(R+r+r \cos v)}+\frac{\mu \cos v}{R+r+r \cos v}
\end{aligned}
$$

In order to solve differential equation (14), some simplification must be done connecting with the practical situation. Multilayer winding drum is usually used for deep mine hoist, and the diameter of the drum is large in deep mine hoist, 


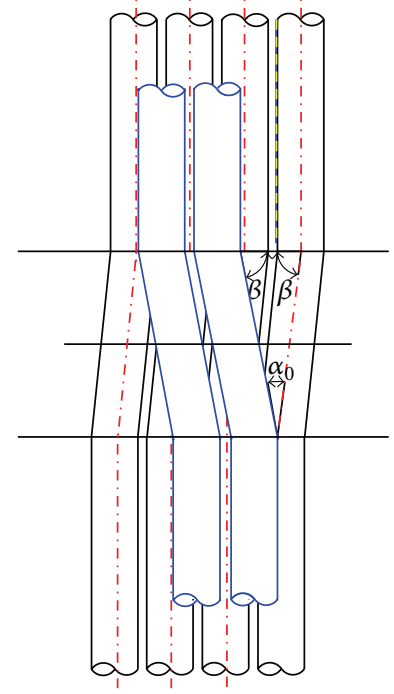

FIGURE 7: The sketch map of the crossover.

and the ratio of the diameter of drum and cable is ordinary 80 , hence the expression

$$
\begin{aligned}
\frac{\cos \alpha \sin v}{R+r+r \cos v} & \approx 0, \\
\frac{\mu \cos v}{R+r+r \cos v} & \approx 0, \\
(R+r+r \cos v) & \approx(R+r) .
\end{aligned}
$$
lows:

Then differential equation (14) can be simplified as fol-

$$
\frac{d \alpha}{d v}=2 \mu \sin \alpha
$$

In order to get the particular solution of the differential equation of the first order, the initial value must be given. The numerical value of relevant parameters can be given based on The South African Bureau of Standards 0294 (2000) [15] such as $d=43 \mathrm{~mm}, \varepsilon=2.5 \mathrm{~mm}$, the helical angle of the crossover cable $\beta$ is close to $\pi / 2$, and the initial value of the parameter $\alpha$ is about $\pi / 90$; that is, $\alpha_{0}=\pi / 90$, as shown in Figure 7 . And the initial value of the polar angle can be calculated through (4); that is, $v_{0}=58 \pi / 180$.

So the particular solution of differential equation can be gotten as follows:

$$
\alpha=2 \arctan \left(\mathrm{e}^{2 \mu \nu-2.02 \mu-4.05}\right) .
$$

The particular solution is substituted into (7) and then obtains

$$
=2 \int_{\arccos ((d+\varepsilon) / 2 d)}^{\pi / 2} \frac{r}{R+r} \frac{1}{\tan \left[2 \arctan \left(\mathrm{e}^{2 \mu \nu-2.02 \mu-4.05}\right)\right]} d v .
$$

Equation (18) is the reasonable theoretical equation of the crossover length; in order to study expediently, definite

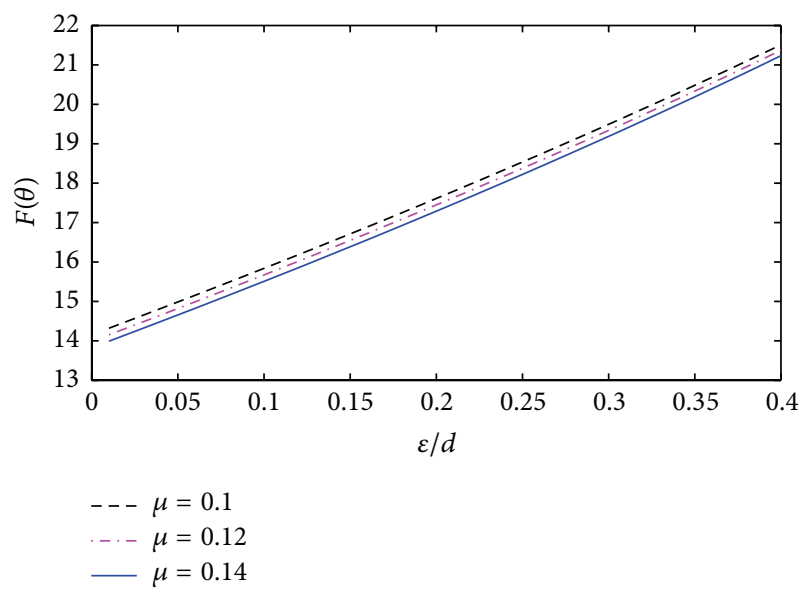

FIGURE 8: The graphics of the functional relationship of $F(\theta)$.

integral is a substitute for $F(\theta)$ as shown in (19), and then (18) is simplified into (20). Consider

$$
\begin{aligned}
F(\theta) & \\
& =\int_{\arccos ((d+\varepsilon) / 2 d)}^{\pi / 2} \frac{1}{\tan \left[2 \arctan \left(\mathrm{e}^{2 \mu \nu-2.02 \mu-4.05}\right)\right]} d v, \\
\gamma & =2 \frac{r}{R+r} F(\theta) .
\end{aligned}
$$

Equation (19) is definite integral and can be calculated by MATLAB programming; the computational result is drawn in curves as shown in Figure 8.

\section{Results and Discussion}

Figure 8 shows that the numerical value of $F(\theta)$ is proportional to the specific value of $\varepsilon / d$, and once the diameter of the drum and the cable is confirmed, the value of $F(\theta)$ is decreased along with the enlargement of the friction coefficient. That is, the larger the friction coefficient is, the smaller the central angle corresponding to the crossover is; the larger the gap of the groove is, the larger the central angle corresponding to the crossover is. Combine Figure 8 with (17); we will find out that the larger the ratio of $r / R$ is, the larger the central angle corresponding to the crossover is.

For explaining the question well, consider an ascending cycle of a deep mine system with the fundamental parameters; that is, $R=1750 \mathrm{~mm}, r=21.5 \mathrm{~mm}, \varepsilon=2.5 \mathrm{~mm}$, and $\mu=0.12$. These parameters are substituted into (18), and the central angle of the crossover arc is $0.35 \mathrm{rad}$. Then based on The South African Bureau of Standards 0294 [16], all half-turn crossovers should be of length 12 times the nominal diameter of the rope, and the central angle of the crossover arc is $0.3 \mathrm{rad}$.

In the second example, Borje of $\mathrm{ABB}$ Mining [17] mentioned that the two crossover sections of the Lebus grooving each span 15 degrees, corresponding to 14 times the rope diameter, and the central angle of the crossover arc is 
$0.344 \mathrm{rad}$. We will find that the result based on our derivation is close to them.

As another example, Kaczmarczyk and Ostachowicz [18] gave the parameters of double-drum Blair multirope system; that is, the winder drum radius is $2140 \mathrm{~mm}$, the cable diameter is $48 \mathrm{~mm}$, and the coil crossover arc is $0.2 \mathrm{rad}$; then the result based on (19), (20) this paper derived should be $0.33 \mathrm{rad}$.

It is not hard to find that the coil crossover arc this paper derived is always bigger than the project data in deep mine hoist. As for why these happen, the parameters that the literature [18] provided come from the Kloof Mine that located in the northern part of South Africa in Gauteng. That means the working condition is always dirty in the real mine hoist systems, and these conditions lead to the friction coefficient being bigger than that under the laboratory conditions. According to (18) that the paper deduced, when the friction coefficient increases, the crossover arc will decrease.

Contrasting this with earlier Lebus liner, Wieschel [19] mentioned that the helical groove portions each comprise an arcuate segment of approximately $45^{\circ}$ of the circumference of the drum. At present, in another Lebus liner, the inclined grooves are about twenty percent of the circumference, that is, $36^{\circ}$. Apparently, the central angle of the crossover arc is bigger than that which this paper derived. It is because the Lebus liner that the literature [19] mentioned mainly for winch and crane and the loading and the drum diameter are relatively small, and the working condition is better than in the mine. In addition, the winch and crane configurate the wire rope with circular strand, and the mine hoist configurates the wire rope with shaped strand, and the friction coefficients of the winch and crane are smaller than the mine hoist, and the drum to rope ratios of the winch and crane are smaller than the mine hoist too. Therefore, the formula this paper derived is only suitable for the mine hoist.

From another point of view, when the cable achieves multilayer winding, the cable would shift the rope loop in the crossover zone; the abrasion and friction in the crossover zone are much severer than in the parallel circular groove and seriously affected the cable's lifetime. Thus, the crossover zone is much longer, the more the cables that would participate the shift in the crossover zone, the more the cables that would wind without groove, and this leads to the cable's instability and irregular arrangement, and this aggravates the wear of the cable and decreases the lifetime of the cable. On the contrary, if the length of the crossover zone is too small, the time that the cable shifts through the crossover zone is too short; it will produce instantaneously shock and greatly shorten the life of the cable. This paper only considers the cable to not slip in the crossover zone. But this paper still gives a better theory reference on the study of multilayer winding in mine hoist.

\section{Conclusions}

In this paper we have established the cable's differential geometry relation in the crossover zones and derived the mathematical expression of the crossover arc. By way of analyzing the differential geometry relation and the equilibrium relationship of the separate forces, the relational expression between geodesic curvature $K_{g}$ and normal curvature $K_{n}$ has been gotten, and the expression is the same as the formula of the fiber stable coiled onto the solid of revolution along the nongeodesic. Then the approximate parametric equation of the first-layer cable has been constituted, and the mathematical expression of $K_{g}$ and $K_{n}$ has been solved, and then the first-order differential equation of the function relationship between $\alpha$ and $v$ has been solved, and we obtain the particular solution. The particular solution is substituted into the mathematical expression of the crossover arc and graphic of the result with MATLAB. Finally, we get the reasonable formula of the central angle of the crossover arc.

The formula this paper derived shows that the central angle of the crossover arc of the deep mine hoist is about the four factors, that is, the diameter of the drum, the diameter of the cable, the friction coefficient of the cable, and the gap of the groove. The relationships among them are shown in (19)(20).

\section{Conflict of Interests}

The authors declare that there is no conflict of interests regarding the publication of this paper.

\section{Acknowledgment}

The study is supported by the National Basic Research Program of China (973 Program 2014CB049403).

\section{References}

[1] C. R. Chaplin, "Failure mechanisms in wire ropes," Engineering Failure Analysis, vol. 2, no. 1, pp. 45-57, 1995.

[2] X.-S. Gong, "The study on multi-layer winding of the mine hoist," Mining Machinery, vol. 12, pp. 7-12, 1985 (Chinese).

[3] X.-S. Gong, Z.-J. Xie, and X. Yang, "Vibration control on winding cable of multiple layers on mine hoists," Journal of Vibration Engineering, vol. 12, no. 4, pp. 460-467, 1999 (Chinese).

[4] S.-J. He, "The motion equations in geodesic winding," Acta Materiae Compositae Sinica, vol. 3, no. 1, pp. 54-60, 1986 (Chinese).

[5] X.-W. Leng, "A General theory of stable non-geodesic winding," Acta Astronautica, vol. 3, pp. 90-99, 1982 (Chinese).

[6] G. Menges, R. Wodicka, and H. L. Barking, "Non-geodesic coiling on a surface of revolution," in Proceedings of the 33rd Annual Conference of SPI, Section 10-D, pp. 1-4, Washington, DC, USA, February 1978.

[7] J. De Carvalho, M. Lossie, D. Vandepitte, and H. Van Brussel, "Optimization of filament-wound parts based on non-geodesic winding," Composites Manufacturing, vol. 6, no. 2, pp. 79-84, 1995.

[8] L. Zu, S. Koussios, and A. Beukers, "Design of filamentwound circular toroidal hydrogen storage vessels based on nongeodesic fiber trajectories," International Journal of Hydrogen Energy, vol. 35, no. 2, pp. 660-670, 2010.

[9] G. M. Wells and K. F. McAnulty, "Computer aided filament coiling using non-geodesic trajectories," in Proceedings of the 6th Conference on Co-mosites Materials, pp. 1.161-1.182, 1987.

[10] J. Scholliers and H. Van Brussel, "Computer-integrated filament winding: computer integrated design, robotic, filament winding 
and robotic quality control," Composites Manufacturing, vol. 5, no. 1, pp. 17-24, 1994.

[11] X.-L. Li and D.-H. Lin, "Non-geodesic winding equations on a general surface of revolution," in Proceedings of the 6th International Conference on Composites Materials, pp. 1.1521.160, 1987.

[12] H.-Y. Fu, K.-B. Huang, F.-Q. Zhu, and D.-D. Han, "Boundary conditions and steady Eq.s for the non-geodesic winding," Journal of Harbin Institute of Technology, vol. 28, no. 2, pp. 125129, 1996 (Chinese).

[13] X.-S. Gong, Z.-J. Xie, and X.-H. Yang, "Vibration control on winding cable of Multiple layers on mine hoists," Journal of Vibration Engineering, vol. 12, no. 4, pp. 460-467, 1999 (Chinese).

[14] A. Gray, Modern Differential Geometry of Curves and Surfaces, CRC Press, Boca Raton, Fla, USA, 1993.

[15] AQSIQ and The China National Standardization Management Committee, Single-Rope Hoist of Mine Winder GB/T 209612007, Standards Press of China, 2007 (Chinese).

[16] The Performance, Operation, Testing and Maintenance of Drum Winders Relating to Rope Safety, 0294, South African Bureau of Standards, 2000.

[17] B. Johansson and A. Steinarson, "A new method for automatic reduction of catenary oscillations in drum hoist installations," in Proceedings of the International Conference on Hoisting and Haulage, pp. 125-139, Hoist \& Haul, 2015.

[18] S. Kaczmarczyk and W. Ostachowicz, "Transient vibration phenomena in deep mine hoisting cables. Part 2: numerical simulation of the dynamic response," Journal of Sound and Vibration, vol. 262, no. 2, pp. 245-289, 2003.

[19] J. E. Wieschel, "Spooling drum including stepped flanges," United States Patent no. 4071205. 


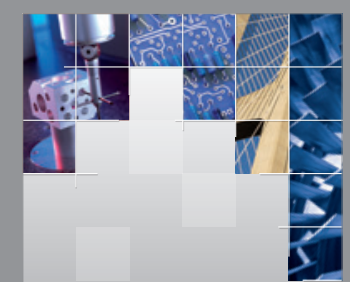

\section{Enfincering}
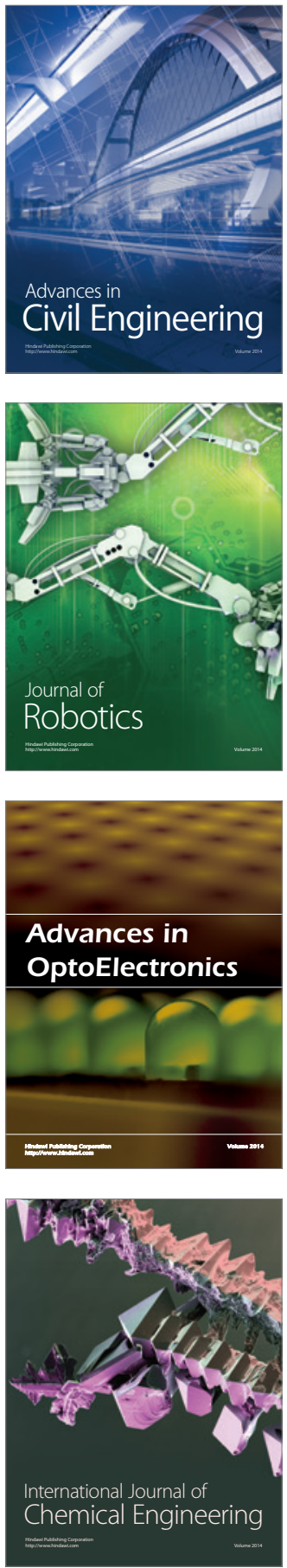

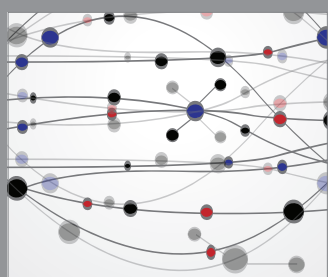

The Scientific World Journal

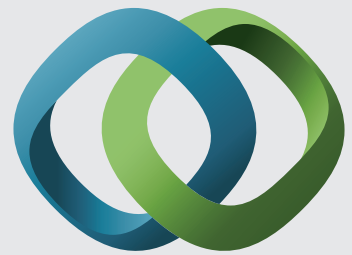

\section{Hindawi}

Submit your manuscripts at

http://www.hindawi.com
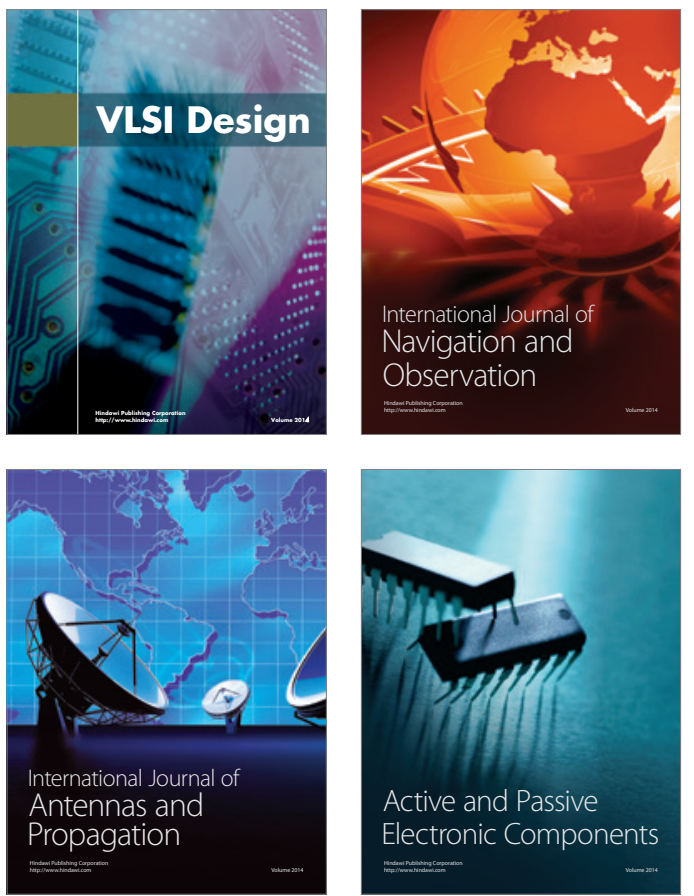
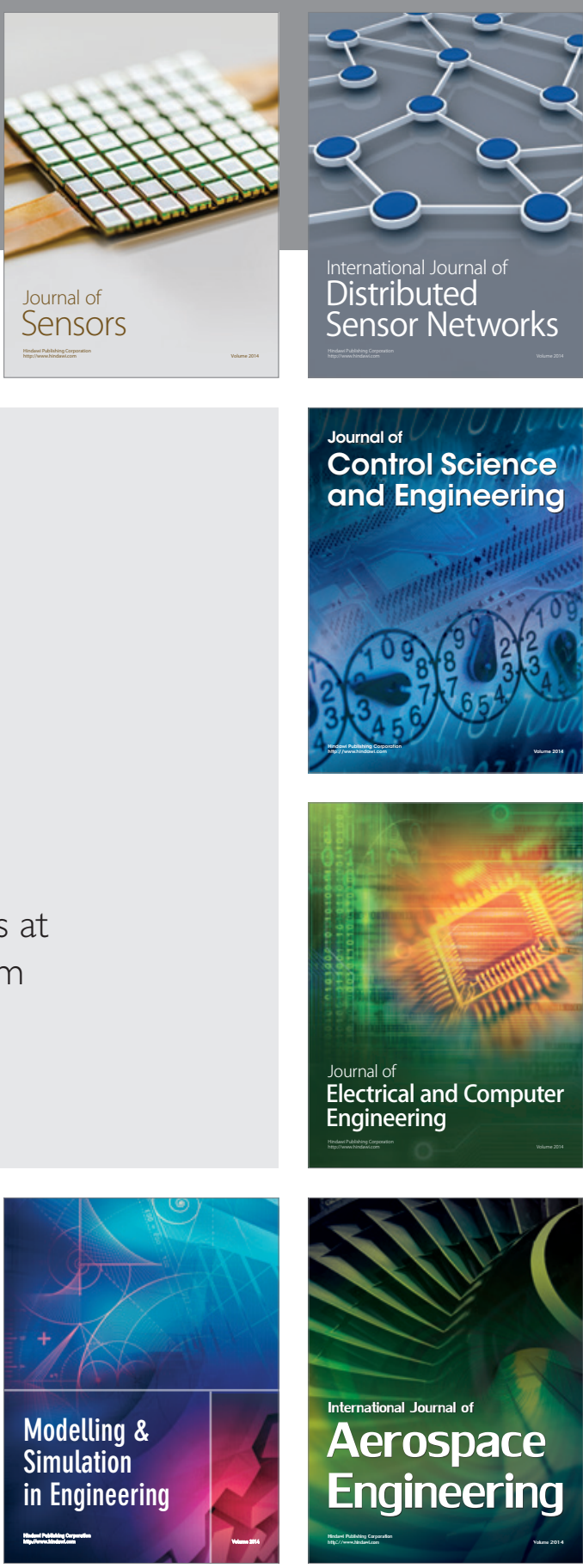

International Journal of

Distributed

Sensor Networks

Journal of

Control Science

and Engineering
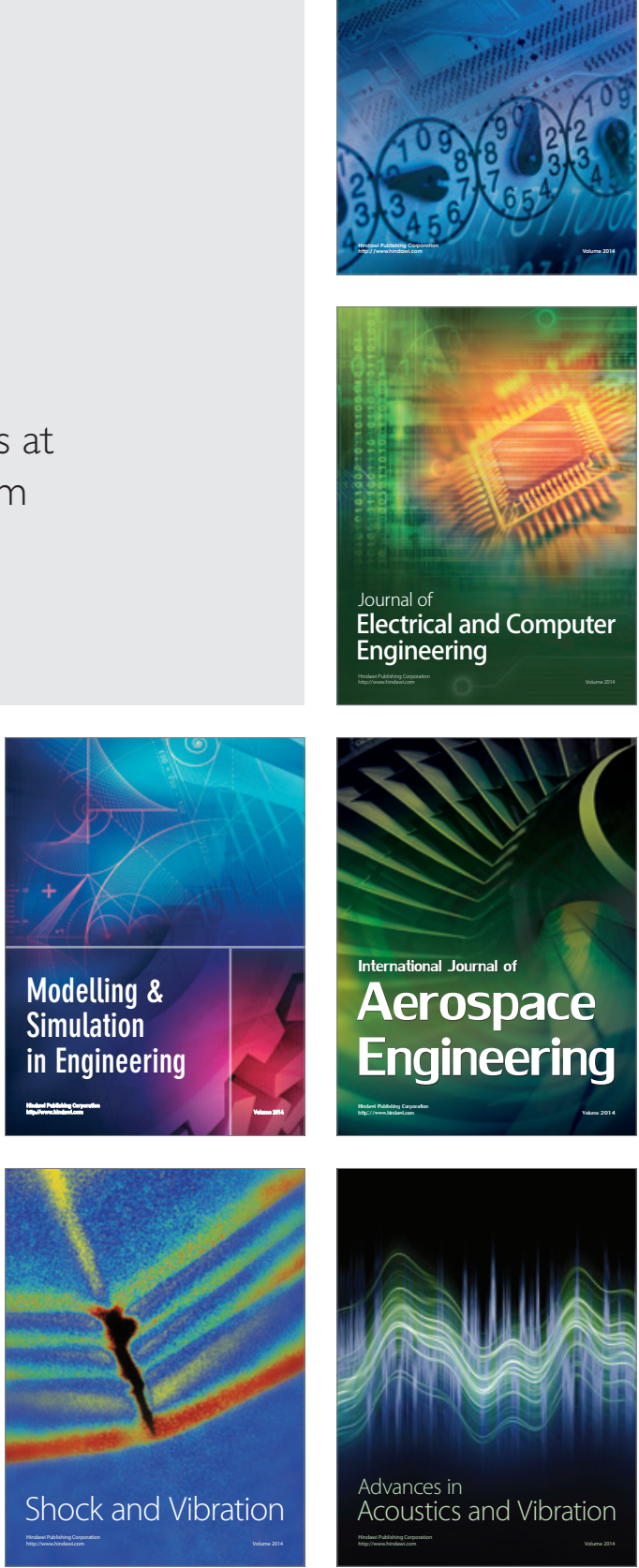